\title{
Changes in left ventricular regional asynchrony after intracoronary thrombolysis in patients with impending myocardial infarction
}

\author{
DEREK GIBSON, $\dagger$ HELMUTH MEHMEL, * FRANZ SCHWARZ, * KUN LI, $\dagger$ \\ WOLFGANG KÜBLER*
}

From the $\star$ University Medical Clinic, Heidelberg, Federal Republic of Germany; and †Brompton Hospital, London

SUMMARY Ventriculograms obtained before and a mean (SD) of $4.3(2.5)$ weeks after intracoronary thrombolysis in 23 patients who were treated within $3.5(3 \cdot 1)$ hours of the onset of pain were examined for changes in asynchronous left ventricular wall motion. Lysis was achieved in 19 patients, and in 16 the affected artery was still patent at restudy. Angiograms were digitised frame by frame. Left ventricular volumes, ejection fraction, and peak ejection rate were all unchanged after thrombolysis, whereas peak filling rate fell, whether or not patency was achieved or maintained. Regional wall motion was examined by means of isometric and contour plots. The area supplied by the affected coronary artery showed simple hypokinesis or akinesis in 10 cases, which was unchanged at the second study in nine and improved in one. The commonest manifestation of asynchrony was delayed inward motion during isovolumic relaxation. This was present in 12 cases with or without associated hypokinesis; after thrombolysis wall motion improved significantly in eight and returned to normal in six, significantly more frequently than it did in patients with simple hypokinesis. Dyskinesis (three patients) and hyperkinesis (five patients) resolved in all. Outward wall motion during isovolumic relaxation reverted to normal in four out of five cases, and outward motion during isovolumic contraction reverted to normal in five out of seven. The frequency of improvement was also increased when the circulation to the affected segment was not compromised by an important residual stenosis. Flow in the affected artery was re-established or maintained significantly less frequently when simple hypokinesis or akinesis was present at the first study. These observations provide further evidence that asynchronous wall motion early after acute myocardial infarction represents residual contractile activity, and suggest that knowledge of its presence and distribution may be useful in assessing patients on whom thrombolysis is performed.

Thrombolysis and other procedures for acute revascularisation can prevent the development of infarction after coronary artery thrombosis in the absence of adequate collateral flow. ${ }^{12}$ During this early period after the onset of symptoms abnormal timing of wall motion is more common than a simple reduction in amplitude. ${ }^{3}$ Asynchronous movement

Requests for reprints to Dr Derek Gibson, Cardiac Department, Brompton Hospital, Fulham Road, London SW3 6HP.

Accepted for publication 10 February 1986 would be expected when the contractile activity of affected myocardium is reduced but not wholly lost. The present investigation was designed to follow the fate of myocardial segments that showed such asynchrony after thrombolysis had been performed, and to compare it with that of the more commonly studied hypokinesis. If asynchrony is indeed the result of a lesser degree of ischaemic injury than that which causes simple hypokinesis, the effects of reestablishing flow might be correspondingly greater. Asynergy might thus be a useful marker for the physiological consequences of thrombolysis in individual patients. 


\section{Patients and methods}

Twenty three patients who fulfilled the criteria for intracoronary thrombolysis were studied by left ventriculography and coronary arteriography at the University Hospital, Heidelberg ${ }^{4}$ within $3.5(1.3)$ hours (mean (SD)) of the onset of pain. Other data from these patients have been included in earlier studies. ${ }^{4-6}$ The patients were premedicated with heparin (7500 units intra-arterially) and prednisone ( $250 \mathrm{mg}$ intravenously). Intracoronary glyceryl trinitrate $(0.2-0.4 \mathrm{mg})$ was administered, and then intracoronary streptokinase $(2000$ units $/ \mathrm{min})$ was given until the patency of the artery was reestablished or until a total dose of 200000 units had been given. Coronary arteriography was repeated every 15 minutes throughout the procedure. The patients were then electively restudied $4.3(2.5)$ weeks later. Left ventriculography was performed in the $30^{\circ}$ right anterior oblique projection with an injection of $40 \mathrm{ml}$ Urografin at a flow rate of $12 \mathrm{ml} / \mathrm{s}$ through a pigtail catheter. The cine film was exposed at 50 frames/s. Calibration was established from movement of the catheter tip positioned in the ventricle when the table was displaced by $8 \mathrm{~cm}$. Extrasystoles and postextrasystoles were not studied. Multiple views of the coronary arteries and vein grafts, if present, were then obtained by Judkins's technique.

\section{ANALYSIS OF ANGIOGRAMS}

Cavity outlines were manually digitised frame by frame over each beat studied ${ }^{7}$ with a fixed external reference point. The timing of aortic valve opening and closure was established by direct inspection, and that of mitral valve opening was taken as occurring on the cine frame which first demonstrated unopacified blood within the left ventricular cavity at the onset of diastole. Ejection was defined as the time between aortic opening and closure, and isovolumic relaxation as the interval between aortic closure and mitral opening. Cavity volume on each frame was derived by the area-length $\operatorname{method}^{8}$ and plotted as a continuous curve, along with its first derivative with respect to time, to give peak rates of ejection and filling. The ejection fraction was calculated as stroke volume divided by end diastolic volume. End diastolic and end systolic volumes, ejection fraction, and peak ejection and filling rates of initial and final ventriculograms were compared in each patient. Two observers (KL and DG) digitised a total of 124 beats, and mean values from at least two beats were used for each determination.

Regional wall movement was demonstrated throughout the cardiac cycle by construction of isometric and contour displays. ${ }^{7}$ The method depends on constructing 40 plots of wall motion against time from 40 points equally spaced around the cavity outline, starting from the junction of the mitral valve with the aortic root. Segments 1-15 represent the mitral valve and inferior wall, segments $15-25$ the apical region, and segments $25-40$ the free wall. The initial and final ventriculograms in each patient were compared with respect to the following features.

Hypokinesis was deemed to be present when the overall amplitude of inward motion between the start of the beat and mitral valve opening was less than the lower $95 \%$ confidence limits for the region in question determined from a series of ventriculograms in 20 patients without demonstrable cardiac disease. Wall motion during ejection was considered in the same way. If the overall amplitude of motion was $\leqslant 2 \mathrm{~mm}$ in either direction, the segment was considered to show akinesis. Dyskinesis was taken to be outward movement of more than $2 \mathrm{~mm}$ that occurred throughout ejection and lasted for at least $100 \mathrm{~ms}$.

Abnormalities of isovolumic contraction-Two types were recognised: outward motion of $\geqslant 2 \mathrm{~mm}$ starting before aortic valve opening and persisting for no more than $60 \mathrm{~ms}$ into ejection, and early inward motion of $\geqslant 3 \mathrm{~mm}$ before aortic valve opening. ${ }^{9}$

Abnormalities of isovolumic relaxation-Again, these were of two types: abnormal inward motion of $\geqslant 2 \mathrm{~mm},{ }^{7}$ and outward motion of $\geqslant 7 \mathrm{~mm}$. The latter abnormality was confined to segments $25-40$ on the free wall.

The extent of each abnormality was determined from the number of segments for which values lay outside the $95 \%$ confidence limits of normal.

\section{Assessment of change in regional motion}

In order to describe any change in local wall motion patients were divided into three groups according to the findings at the initial angiogram: (a) those in whom the region of ventricle supplied by the thrombosed artery showed hypokinesis, akinesis, or dyskinesis only, a pattern of motion referred to as simple hypokinesis; (b) those in whom the affected region showed overall hypokinesis or dyskinesis, but in addition, there was abnormal inward wall motion during isovolumic relaxation; and (c) those in whom the affected region showed normal overall amplitude of motion, but in whom there was abnormal inward motion during isovolumic relaxation.

In each of these groups, regional wall motion was assessed in terms of amplitude and in terms of the four disturbances affecting the isovolumic periods. Based on previous studies of the reproducibility of the method of angiographic analysis, ${ }^{10}$ changes in regional amplitude of motion were regarded as significant when their extent around the cavity outline between the two angiograms differed by more 
than four segments (that is by more than $10 \%$ ), and when values moved from outside to inside normal $95 \%$ limits or vice versa, the former being considered an improvement and the latter a deterioration. Changes in wall motion during the two isovolumic periods were more complex to assess, since such asynchrony was regarded as being intermediate between normality and complete disappearance of function. ${ }^{3}$ Its loss might thus be a sign of either recovery or infarction. In order to resolve this difficulty, changes in the timing of wall motion were considered in conjunction with those of amplitude at the time of the second study. Only if amplitude was normal when asynchrony had resolved, was the change regarded as an improvement. Loss of asynchrony in the setting of persistent hypokinesis or akinesis was taken to represent part of the natural history of unmodified myocardial infarction.

\section{ANALYSIS OF CORONARY ARTERIOGRAMS}

Coronary arteriograms obtained during the first study before thrombolysis was performed were examined to determine which vessels were obstructed by thrombus and whether the other two main arteries were diseased. Collateral vessels were sought, and, when present, opacification of the occluded vessel itself was distinguished from that of its branches only. Arteriograms from the second study were examined to determine the state of the vessel in which flow was initially re-established and the presence, approximate severity of any underlying fixed stenosis, and, when present, a vein graft were noted. The state of the other vessels was also reassessed. In view of variability in the subjective analysis of coronary arteriograms, ${ }^{11}$ stenoses were recorded as being mild or severe, with no attempt to quantify them in terms of percentage narrowing of the lumen. From this information, the affected artery was categorised as: $(a)$ reoccluded or unchanged; $(b)$ patent, but with a severe proximal stenosis, or $(c)$ normal, with either a mild proximal stenosis or a functioning vein graft. Serum creatine concentration was measured ${ }^{6}$ and peak values were noted.
STATISTICAL METHODS

A paired Student's $t$ test was used to compare differences between means. Differences between groups in the frequency of a single abnormality were examined by Fisher's exact probability test.

\section{Results}

\section{CORONARY ARTERIOGRAPHY}

In 19 out of the 23 cases flow was re-established in one of the three coronary arteries. In eight cases the right coronary artery was involved; three of these patients had important additional disease of one or more of the other two arteries. In four cases the circumflex artery was affected by thrombosis with other important disease in two cases, and in seven cases the anterior descending artery was thrombosed with additional disease in four. At the second study the thrombosed vessel remained patent in 16 cases, whereas in three (one right, one anterior descending, and one circumflex artery) it had occluded again. Collaterals were identified in seven cases; the distal segment of the occluded vessel was filled in five and branches only in two.

Examination of the arterial supply to the affected region at the second study showed that in six cases the situation was unchanged from that found on the first occasion, either because of unsuccessful thrombolysis or because of reocclusion (three cases each). In 11 cases there was an important proximal stenosis, and in six the arterial supply was uncompromised, either because there was a functioning vein graft (four cases) or because there was no important proximal stenosis (two cases).

\section{OVERALL LEFT VENTRICULAR FUNCTION}

The Table gives details of the results. There was no significant difference between the two studies for values of end diastolic or end systolic volume, ejection fraction, or peak ejection rate, although there was a consistent fall in peak filling rate by $160 \mathrm{ml} / \mathrm{s}$ $(p<0.05)$. Similar results were obtained when the

Table Overall left ventricular function before and after thrombolysis

\begin{tabular}{|c|c|c|c|c|c|c|c|}
\hline & \multirow[t]{2}{*}{ Normal } & \multicolumn{2}{|c|}{ All patients } & \multicolumn{2}{|c|}{ Improved flow } & \multicolumn{2}{|c|}{ Flow unchanged } \\
\hline & & Before & After & Before & After & Before & After \\
\hline $\begin{array}{l}\text { End diastolic volume }(\mathrm{ml}) \\
\text { End systolic volume }(\mathrm{ml}) \\
\text { Ejection fraction }(\%) \\
\text { Peak ejection rate }(\mathrm{ml} / \mathrm{s}) \\
\text { Peak filling rate }(\mathrm{ml} / \mathrm{s})\end{array}$ & $\begin{array}{l}150(35) \\
30(15) \\
65(7) \\
580(210) \\
690(260)\end{array}$ & $\begin{array}{l}180(55) \\
60(35) \\
62(12) \\
680(200) \\
670(220)\end{array}$ & $\begin{array}{l}170(50) \\
60(40) \\
66(15) \\
590(240) \\
510(200)^{\star}\end{array}$ & $\begin{array}{l}170(50) \\
65(35) \\
62(9) \\
690(190) \\
660(250)\end{array}$ & $\begin{array}{l}160(50) \\
50(80) \\
69(13) \\
640(230) \\
540(210)^{\star}\end{array}$ & $\begin{array}{l}200(75) \\
90(70) \\
57(17) \\
730(270) \\
740(220)\end{array}$ & $\begin{array}{c}190(40) \\
85(50) \\
58(18) \\
540(250) \\
480(160)\end{array}$ \\
\hline
\end{tabular}

${ }^{\star} \mathrm{p}<0.05$ for change before and after thrombolysis. 

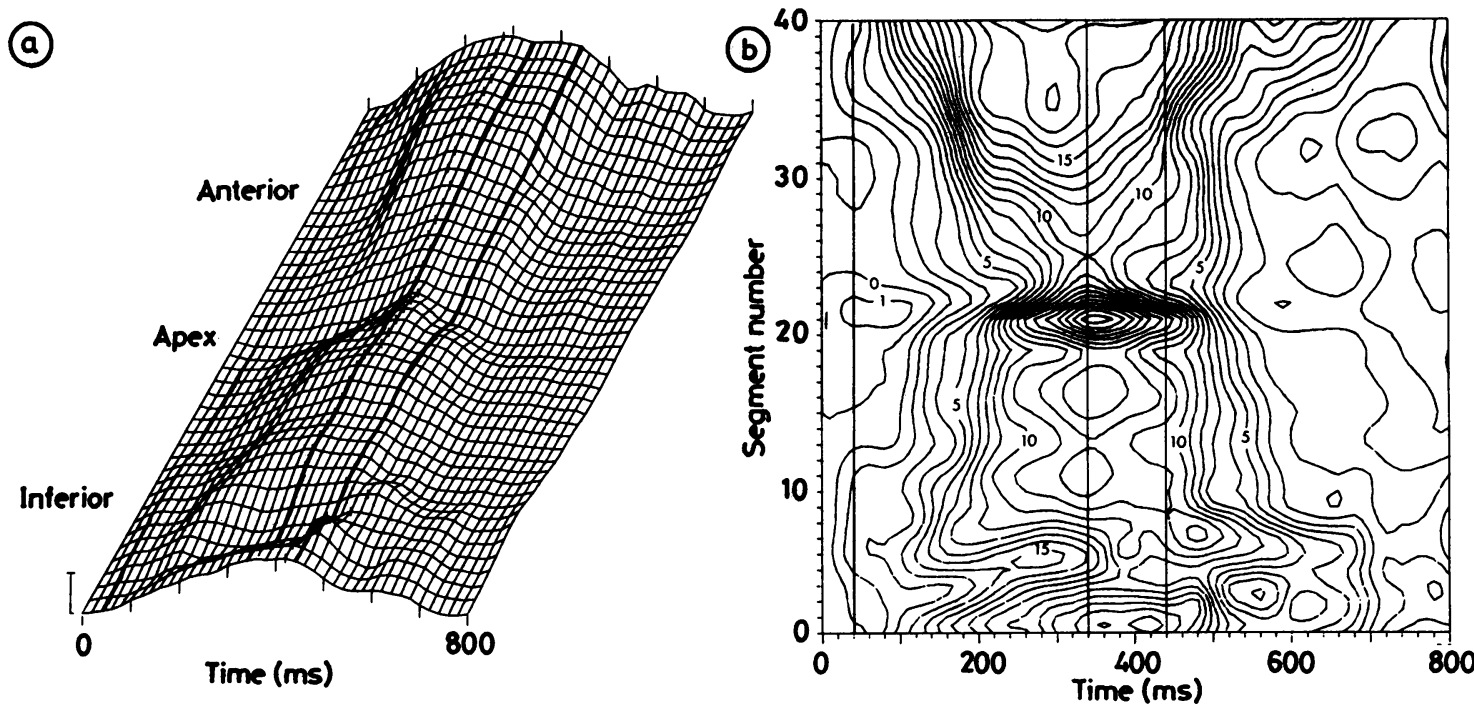

Fig. 1 Isometric (a) and contour (b) displays of regional left ventricular wall motion when left ventricular function is normal. Accentuated time markers (isochrones) represent aortic valve opening, aortic valve closure, and mitral valve opening. For description see text.

subgroup of patients in whom flow was reestablished was considered separately, with the mean value of peak filling rate falling by $120 \mathrm{ml} / \mathrm{s}$ $(p<0.05)$. Patients with values outside the normal $95 \%$ confidence limit of normal at the first study were also considered separately. End diastolic volume was significantly increased in seven patients. It fell in six and was unchanged in one, the mean change being $-50(30) \mathrm{ml}(\mathrm{p}<0.05)$. End systolic volume was increased in eight patients, and fell by a mean of $25(32) \mathrm{ml}(\mathrm{p}<0.05)$. There was no significant change in ejection fraction in the subgroup of patients in whom values were reduced at the first investigation, whereas the $95 \%$ confidence limits of peak ejection and filling rate were so wide that all values fell within the normal range at the first examination.

\section{REGIONAL WALL MOVEMENT}

Normal. Figure 1 gives examples of isometric and contour displays of the left ventriculogram of a normal subject. On the isometric display (Fig. 1a) the horizontal lines represent the 40 plots of wall motion against time, inward displacement being represented as a curve upwards. Their origin from around the cavity outline is shown on the left. The diagonal lines are isochrones, joining events occurring simultaneously on each plot, one for each cine frame. The accentuated isochrones correspond to aortic opening, aortic closure, and mitral opening. The contour display is derived from the same information. Each contour represents $1 \mathrm{~mm}$ of inward or outward movement of the endocardium from its position in the end diastolic frame. The three vertical lines again correspond to aortic opening and closure and mitral opening. In the normal subject there is no appreciable wall motion before aortic opening. During ejection, there is synchronous inward motion, as shown by the contour lines being almost vertical except for a small region at the apex. On the free wall, outward motion starts before mitral opening, and precedes that at the apex or along the inferior wall. After mitral opening, outward motion is rapid and synchronous, as shown by closely spaced and vertical contours.

Simple hypokinesis. In six patients in whom thrombolysis was achieved, and in whom the artery remained patent, simple hypokinesis was demonstrated at the first study in the region supplied by the thrombosed artery (four anterior descending, one right, and one circumflex artery). It remained unchanged at the second study in all. Figure 2 shows isometric and contour plots from a patient with a region of simple hypokinesis on the inferior wall between segments 10 and 15 . The amplitude of inward motion is reduced to $4 \mathrm{~mm}$. Its onset is delayed, but there is no significant movement in either of the two isovolumic periods. Motion in the rest of the cavity outline is normal, both with respect to amplitude and timing. The pattern at the later study (Fig. 2) is virtually unchanged. A minor diminution in amplitude on the anterior wall was noted, 
(a)
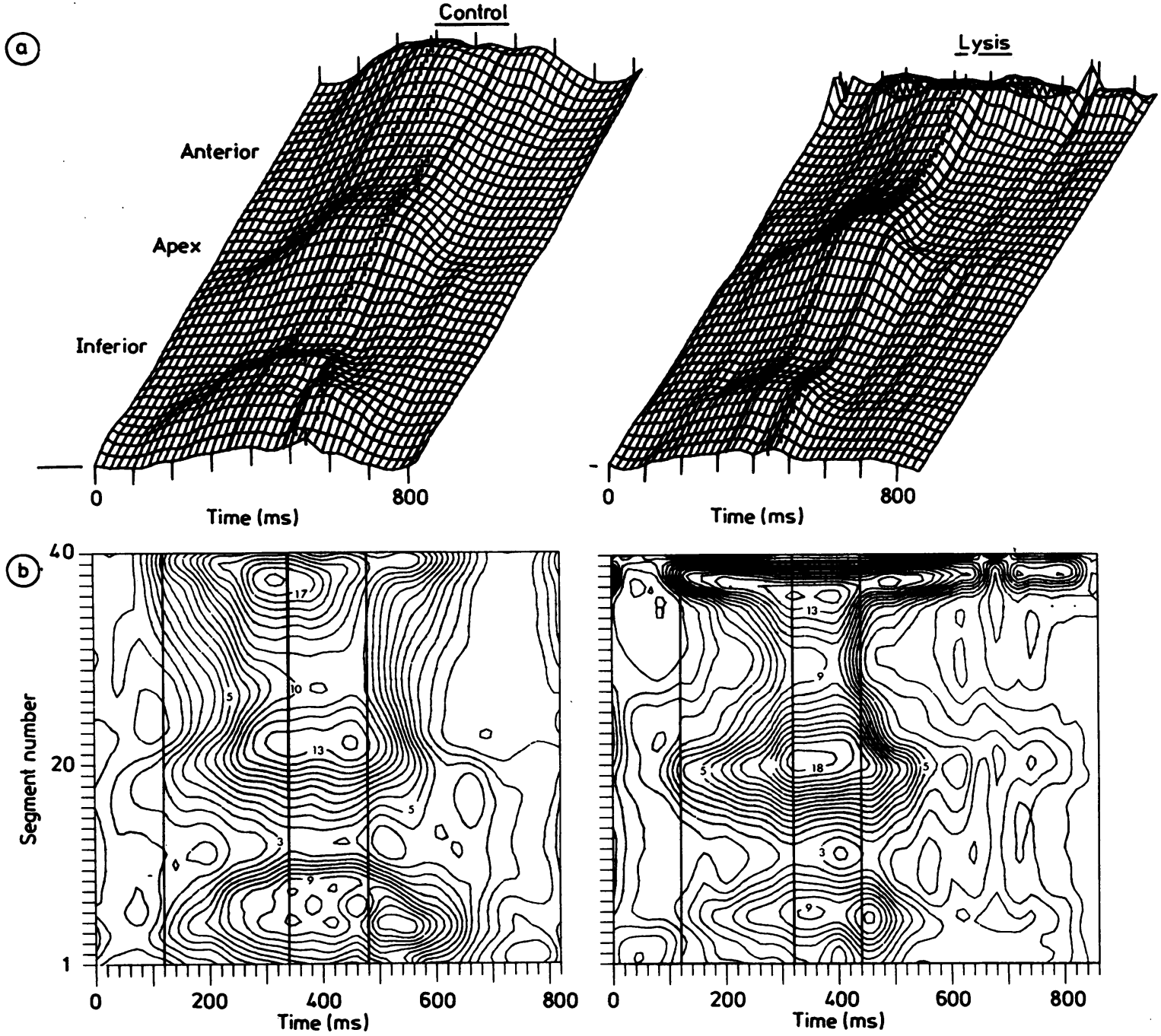

Fig. 2 Isometric and contour displays of left ventriculograms before (left hand isometric and contour displays) and after (right hand isometric and contour displays) thrombolysis from a patient with thrombosis of the circumflex coronary artery. There was a region of simple hypokinesis on the inferior wall which was unchanged at the second examination. For full discussion see text.

but this remained within normal $95 \%$ confidence intervals. Similar regions of simple hypokinesis were also unaltered in three cases in which the recanalised artery was occluded at the second study, and in two in whom thrombolysis had initially been unsuccessful. In one case in whom thrombolysis had been unsuccessful, but in whom a major stenosis of the anterior descending artery had been bypassed, the free wall (segments 20-30), which had been akinetic at the first study, returned to normal although this was associated with the development of abnormal inward movement during isovolumic relaxation on the inferior wall (segments 8-20). Thus simple hypokinesis was present initially in 11 cases; it persisted unchanged in 10 despite successful thrombolysis in six, and was improved but did not return to normal in one with a vein graft.

Hypokinesis associated with delayed inward motion during isovolumic relaxation. This combination of abnormalities was present in six patients, three with 


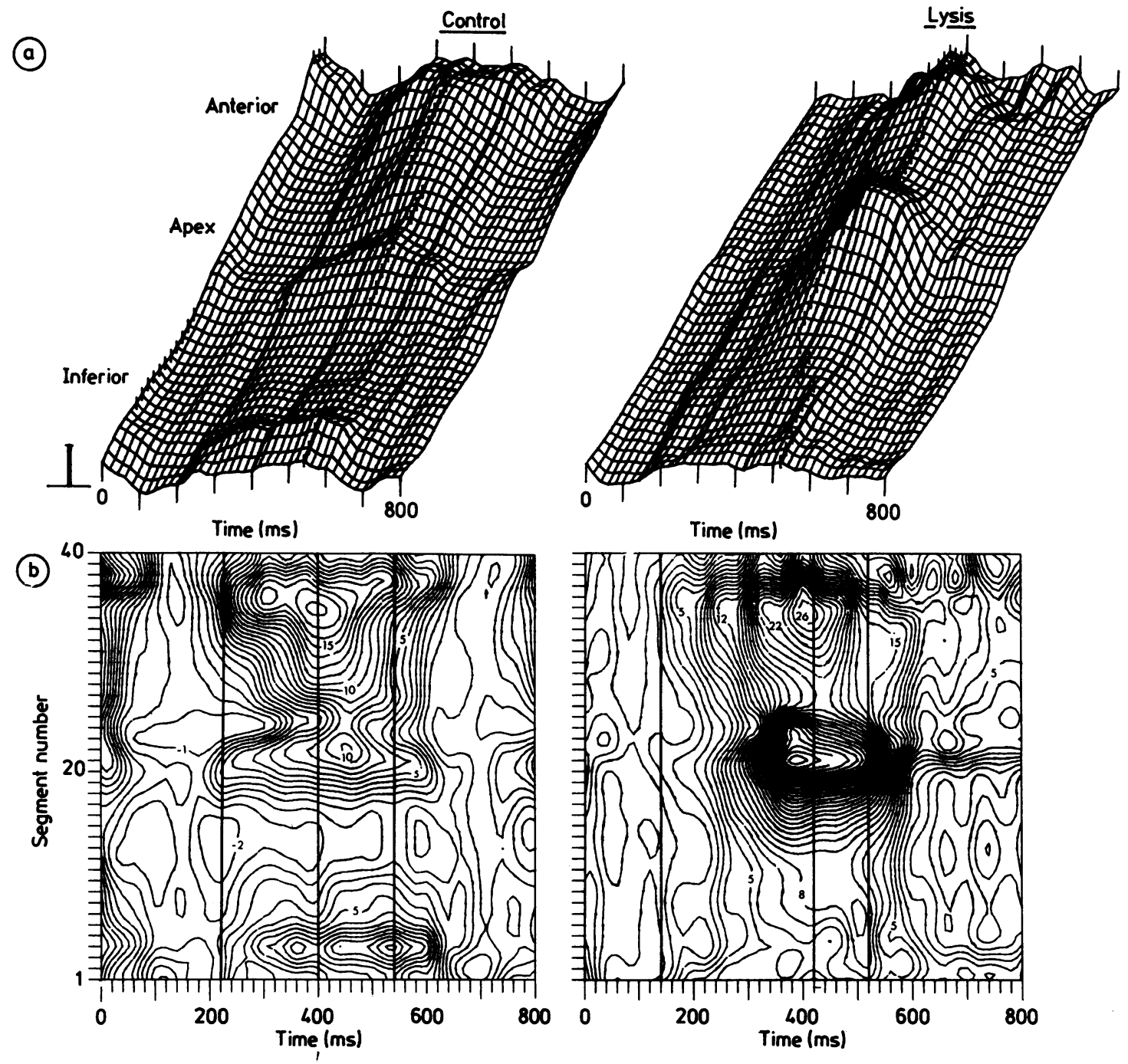

Fig. 3 Isometric and contour displays from a patient with thrombosis of the right coronary artery, in whom the control angiogram showed hypokinesis associated with delayed inward movement of the inferior wall. There was substantial improvement after thrombolysis. Layout as in Fig. 2. For full discussion see text.

disease of the right and three with disease of the anterior descending artery. Figure 3 shows results from a patient with right coronary artery thrombosis. In the first study (Fig. 3) there was a hypokinetic segment on the inferior wall, which showed a total inward motion of $4 \mathrm{~mm}$, less than the normal lower $95 \%$ confidence interval, of which $2 \mathrm{~mm}$ occurs during isovolumic relaxation associated with a $10 \mathrm{~mm}$ early outward motion ${ }^{12}$ along the free wall. During isovolumic contraction there is $2 \mathrm{~mm}$ abnormal outward movement along the inferior wall, associated with premature inward movement before aor- tic valve opening on the free wall. At the second study (Fig. 3) the amplitude of movement in the inferior region had returned to normal. There was still some inward motion during isovolumic relaxation, but this was less extensive than in the earlier study and was almost completely confined to the region of the mitral valve. Wall movement during isovolumic contraction had become completely normal. There had thus been considerable improvement in regional wall motion in the inferior segment that was supplied by the affected coronary artery. In a second patient, who also had right coronary artery 


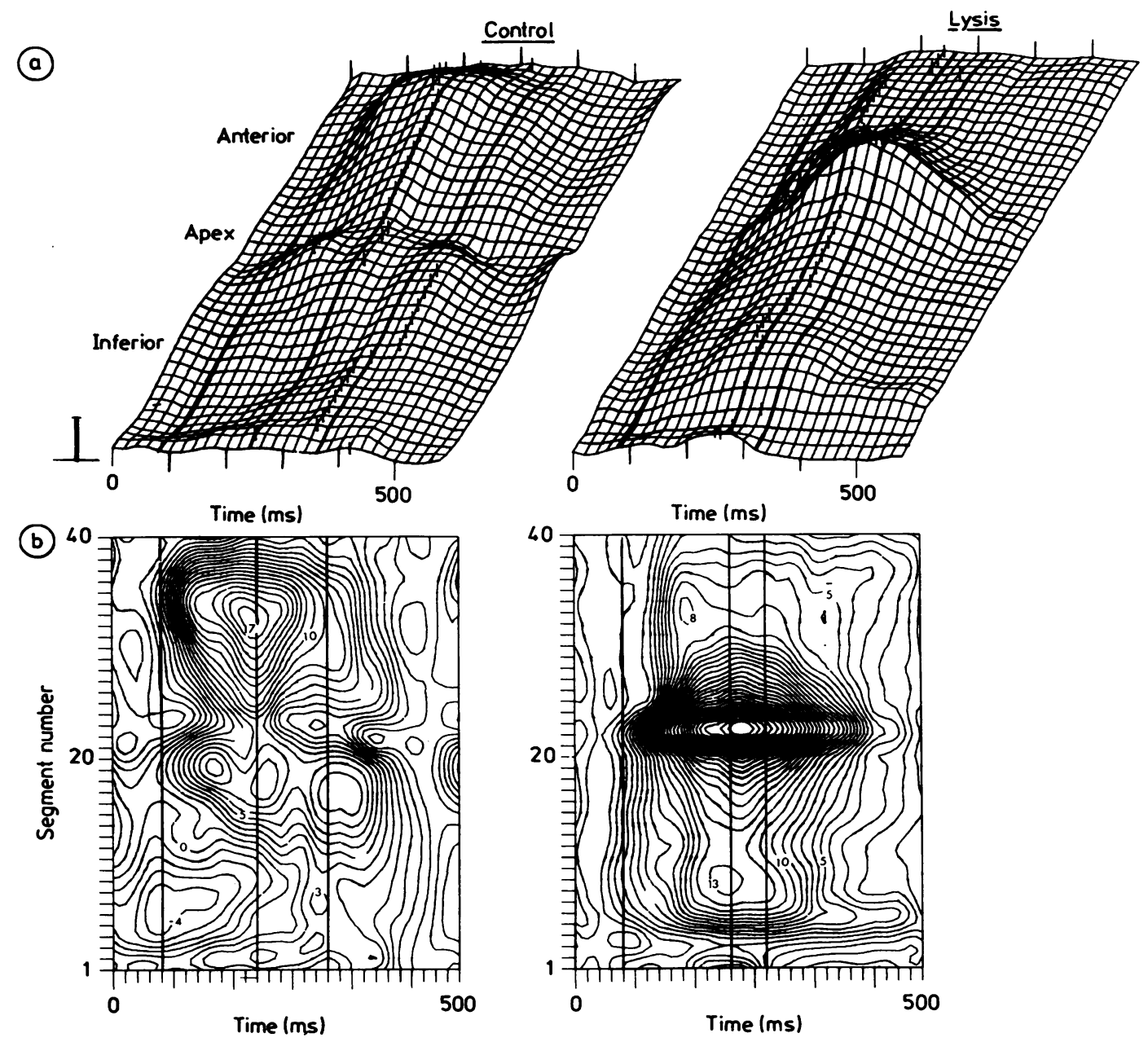

Fig. 4 Isometric and contour displays from a patient with circumflex coronary thrombosis showing delayed inward motion on the inferior wall and early outward motion on the free wall. Both these abnormalities have improved after thrombolysis. Layout as in Fig. 2. For full discussion see text.

disease, wall motion in the affected area returned completely to normal; the amplitude of motion had increased and the disturbance of wall motion during isovolumic relaxation was no longer apparent. One case, with anterior descending thrombosis, improved, with $3 \mathrm{~mm}$ of dyskinesia being replaced by $4 \mathrm{~mm}$ of inward motion. In three cases, the extent and distribution of the hypokinesis were unchanged, though inward movement during isovolumic relaxation was no longer apparent.

Inward motion during isovolumic relaxation with normal amplitude of wall motion. This pattern of wall motion was seen in eight patients, four with right, one with anterior descending, and three with circumflex artery disease. In six the amplitude was normal over the whole segment, and in two over part of that showing asynchrony. Figure 4 shows the results from one of those with circumflex thrombosis. The earlier study (Fig. 4) demonstrated delayed inward motion in segments 5-12 along the inferior wall during isovolumic relaxation, though the total amplitude of inward movement was normal. At the same time, there was early outward movement along the free wall associated with hyperkinesis during systole. There was also early outward movement during isovolumic contraction in segments 4-9. Direct inspection of the two displays shows that movement of the inferior part of the ventricle over 
segments 1-20 was delayed with respect to the free wall by approximately $150 \mathrm{~ms}$ throughout the whole cardiac cycle. After successful thrombolysis, the pattern of wall motion had become entirely normal in amplitude and timing. This response was seen in four further patients (two with thrombosis of the circumflex artery and two with thrombosis of the right coronary artery), but in the other three the abnormality of isovolumic relaxation persisted. Thus in 12 patients the affected area of myocardium showed delayed inward motion during isovolumic relaxation, with or without hypokinesis, which improved significantly in eight and reverted completely to normal in six. The effect of thrombolysis on segments with this pattern of motion thus differs significantly from that on segments showing simple hypokinesis $(\mathrm{p}<0.010)$.

Additional abnormalities of wall motion amplitude.-Hyperkinesis was present in five patients at the time of the first study and dyskinesis in three (in two as an isolated abnormality and in one in association with abnormal inward motion during isovolumic relaxation). Neither abnormality was present in any patient at the time of the second study.

Additional abnormalities of wall motion during the two isovolumic periods.-Additional abnormalities of wall motion during the two isovolumic periods were also considered in association with the amplitude of wall motion in the region supplied by the affected coronary artery. Early outward wall motion during isovolumic relaxation reverted to normal in all of five patients who initially had thrombosis of the right or circumflex coronary artery; in four this was associated with a normal amplitude of wall motion in the inferior region and in one with akinesis. It appeared de novo in the second study in a patient in whom the affected vessel had become reoccluded and in whom akinesis persisted in the region supplied by the occluded vessel. In seven patients (four with thrombosis of the right coronary artery and three with thrombosis of the circumflex coronary artery) there was early outward motion along the inferior wall during isovolumic contraction. In five it disappeared as overall amplitude of motion in the affected region returned to normal. In two, in whom amplitude also remained unchanged, it did not. Early inward movement along the free wall during isovolumic contraction was seen only in those patients with simultaneous outward motion on the inferior wall. It returned to normal after thrombolysis in all but one case.

Relation of changes in wall motion to peripheral arterial supply. - In six patients the arterial supply to the affected region of ventricle was essentially unchanged at the second examination, either because of failure of thrombolysis or because of reocclusion.
In five of these patients, hypokinesis was present at the initial examination, with or without evidence of asynchrony, and this feature persisted, unchanged in distribution, at the second examination. In one case an isolated abnormality of isovolumic contraction returned to normal. Thus one out of six cases showed improvement. In 11 patients blood supply to the affected region was restored at the second examination but there was a major proximal stenosis. In four of these the amplitude of movement returned to normal, but in three asynchrony persisted (one in isovolumic contraction and two in isovolumic relaxation). In the remainder, hypokinesis persisted unchanged, with loss of asynchrony in two. In this group, therefore, appreciable improvement was seen in four, while seven remained unchanged. In five patients a normal or virtually normal arterial supply was restored. There was considerable improvement in wall motion in all. In two, hypokinesis associated with asynchrony became completely normal, in two isolated asynchrony became normal, and in one isolated hypokinesis was converted to normal amplitude but asynchrony developed. These differences in the incidence of improvement in relation to blood supply were statistically significant $(p<0.007)$. Changes in the pattern of wall motion were also related to the peak serum creatine kinase concentrations recorded during the acute illness. In the patients in whom there had been no change in wall motion at the second study, peak serum creatine kinase was $2010(1450) \mathrm{U} / 1$. This was significantly greater than in patients in whom improvement was seen $(1290(800) U / 1(p<0.05))$ and in those in whom wall motion became normal $(810(510) \mathrm{U} / 1$ $(p<0.01))$. No aspect of regional wall motion could be related to the presence or absence of collateral vessels at the first study or to the time after the onset of symptoms at which reperfusion was achieved.

Relation between wall motion and the first study and success of thrombolysis. - Thrombolysis was unsuccessful in seven patients either because flow was not re-established (four cases) or because the vessel had become reoccluded by the second study (three cases). In six of these cases, there was hypokinesis with no evidence of asynchrony. In the remaining cases in which thrombolysis was successful and the vessel remained patent at the second study, ${ }^{13}$ there was asynchrony during isovolumic contraction or relaxation in 11. This difference is significant $(p<0.025)$.

\section{Discussion}

The aim of thrombolysis is to re-establish flow to ischaemic regions of the ventricle and so to preserve myocardial function that would otherwise be lost. If 
the procedure is to be of any clinical value it must improve left ventricular function. Such an improvement may be difficult to document because measurements before and after thrombolysis are made under widely different physiological conditions. Impending infarction is likely to be associated with increased autonomic activity resulting from various stimuli both cardiovascular and psychogenic. These affect cardiac output, heart rate, inotropic state, and peripheral impedence to which all commonly used measures of left ventricular function are sensitive. Differences in the pattern of left ventricular wall motion between the two studies must therefore be interpreted with caution, particularly when changes within the normal range occur.

The effect of thrombolysis on ejection fraction has been widely studied. The lack of change previously reported in the present group of patients ${ }^{4}$ was confirmed in later studies. ${ }^{14-16}$ Other responses of ejection fraction to thrombolysis have been described, including an increase conditional upon the presence of collateral vessels, ${ }^{13}{ }^{17}$ or a consistent increase $^{1819}$ seen even when treatment is delayed by up to 18 hours after the onset of symptoms. One possible reason for ejection fraction failing to rise is that impaired function in one region of the ventricle is often accompanied by hyperkinesis elsewhere, which, in common with others, ${ }^{1520}$ we found at the second study had regressed. Normal values in the acute phase and the frequent occurrence of spontaneous change ${ }^{21}$ were further reasons for ejection fraction being an insensitive index of the effects of thrombolysis. Other measures of overall left ventricular function were no more helpful. End diastolic and end systolic volumes were usually within the normal range, although they did tend to return towards normal if they were initially increased. Peak ejection rate was unchanged between the two studies, while the peak filling rate tended to fall, whether or not patency was achieved or maintained. We conclude that ejection fraction and other measures of overall left ventricular function are of only limited value for following the effects of thrombolysis.

Striking changes in regional wall motion occurred after thrombolysis; these depended on the pattern present at the initial study. After thrombolysis segments showing asynchrony were more likely to return to normal motion than those showing simple hypokinesis. Even within the group of patients with asynchrony, improvement was more likely if overall amplitude was initially normal rather than reduced. These differences in the incidence of improvement suggest a hierarchy of levels of impairment of ventricular function that are reflected in wall movement. It appears that ischaemic injury first shows itself as asynchrony rather than any change in amplitude.
Overall amplitude drops only when the injury is more severe, although even at this stage asynchrony may persist as evidence of residual contractile activity, showing itself most characteristically as inward movement during isovolumic relaxation. Segments showing simple hypokinesis, akinesis, or even dyskinesis if the free wall of the ventricle is affected, have suffered a degree of injury from which they are unlikely to recover even if the circulation is restored. Analysis of regional movement, therefore, can indicate segments which have undergone a comparable degree of injury, and the factors affecting the extent of recovery can be identified. Of these the most important seemed to be the extent to which flow to the affected region had been re-established, since wall motion was much more likely to return to normal when the arterial supply was uncompromised. We did not find the same absolute dependence of improvement on vein grafting reported by Sheehan et al, ${ }^{22}$ though the general principle of ensuring an adequate blood supply to affected areas was confirmed. A second factor was the peak level of serum creatine kinase activity, but there was no relation between the frequency recovery and the time from onset of symptoms to re-establishment of flow. Somewhat unexpectedly the presence of simple hypokinesis at the initial study proved to be a predictor of whether thrombolysis would be successful. This finding suggests that when myocardial function is irreversibly damaged, the integrity of the microcirculation itself is lost, so that even when the thrombus is lysed peripheral runoff is usually inadequate to maintain patency. Such a relation between initial wall motion and subsequent outcome of thrombolysis would invalidate the use of patients in whom lysis was not achieved as controls for assessment of the effects of the procedure on the left ventricular function in those in whom it was successful. Finally, these conclusions are not comparable with those based on the two frame method of analysis of regional wall motion. In this technique single frames corresponding to end systole and end diastole are identified so that regions showing asynchrony would be classified as either normal or hypokinetic depending on the frame chosen as "end systolic.""23 Similarly, regions showing asynchrony with reduced overall amplitude would not be distinguished from those showing simple hypokinesis by this method. Our results are thus not comparable with those in which wall motion is analysed in terms of regional ejection fraction, ${ }^{4}$ hemichord shortening, ${ }^{142425}$ or the "center line" technique, ${ }^{20}$ all of which are based on observations of only two frames. Rather, our results indicate the potential advantages of distinguishing abnormalities of amplitude from those of timing, which can only be achieved by considering 
all the frames in the cardiac cycle.

The present study was an exploratory one. It was retrospective and contained no control group to allow for the effect of spontaneous recanalisation or changes in ventricular function occurring between the two studies. It does not therefore confirm or refute the therapeutic effectiveness of thrombolysis. It does, however, suggest that a more elaborate analysis of regional wall motion than is usually undertaken may be helpful in such cases, in which a major objective is to detect and salvage compromised myocardium. If asynchrony is the hallmark of such areas, ability to locate it and to follow its progress might have practical consequences in determining therapeutic strategy in individual patients. It might be possible on the basis of the pattern of regional wall motion to exclude cases in whom success was unlikely, to identify those in whom myocardium was potentially salvagable even more than six hours after the onset of symptoms, or to follow the effects of less elaborate procedures such as intravenous thrombolysis. This information is potentially available non-invasively by digital subtraction or radionuclide angiography, and to a more limited extent by cross sectional echocardiography. Such imaging methods in combination with automatic boundary recognition and image analysis might provide a means by which these aims could be advanced.

\section{References}

1 Leffel GL, Braunwald E. Thrombolytic therapy. A new strategy for the treatment of acute myocardial infarction. N Engl f Med 1984;311:710-7.

2 Rentrop KP, Cohen M, Hosat ST. Thrombolytic therapy in acute myocardial infarction: review of clinical trials. $A m \mathcal{F}$ Cardiol 1984;54:E-29-31.

3 Gibson D, Mehmel H, Schwarz F, Li K, Kübler W. Asynchronous left ventricular wall motion early after coronary thrombosis. Br Heart f 1986;55:4-13.

4 Schwarz F, Schuler G, Katus H, et al. Intracoronary thrombolysis in acute myocardial infarction: correlations among serum enzymes, scintigraphic and hemodynamic findings. Am $\mathcal{f}$ Cardiol 1982;50:32-8.

5 Schuler G, Schwarz F, Hofmann M, et al. Thrombolysis in acute myocardial infarction using intracoronary streptokinase: assessment by thallium-201 scintigraphy. Circulation 1982;66:658-64.

6 Schwarz F, Faure A, Katus H, et al. Intracoronary thrombolysis in acute myocardial infarction: an attempt to quantitate its effect by comparison of enzymatic estimate of myocardial necrosis with left ventricular ejection fraction. $A m \mathcal{F}$ Cardiol 1983;51:1573-8.

7 Gibson DG, Prewitt TA, Brown DJ. Analysis of left ventricular wall movement during isovolumic relaxation and its relation to coronary artery disease. Br Heart f 1976;38:1010-9.
8 Dodge HT, Sandler H, Baxley WA, Hawley RR. Usefulness and limitations of radiographic methods for determining left ventricular volume. $A m \mathcal{F}$ Cardiol 1966;18:10-24.

9 Gibson DG, Fleck E, Rudolph W. Effect of postextrasystolic potentiation on amplitude and timing of regional left ventricular wall motion in ischaemic heart disease. Br Heart $\mathcal{f}$ 1983;49:466-76.

10 Gibson DG, Doran JH, Traill TA, Brown DJ. Abnormal left ventricular wall movement during early systole in patients with angina pectoris. Br Heart $\mathcal{f}$ 1978;40:758-66.

11 DeRouen TA, Murray JA, Owen W. Variability in the analysis of coronary arteriograms. Circulation 1977;55:324-8.

12 Altieri PI, Wilt SM, Leighton RF. Left ventricular wall motion during the isovolumic relaxation period. Circulation 1973;48:499-505.

13 Rentrop P, Smith H, Painter L, Holt J. Changes in left ventricular ejection fraction after intracoronary thrombolytic therapy. Circulation 1983;68:I-55-60.

14 Ritchie JL, Davis KB, Williams DL, Caldwell J, Kennedy JW. Global and regional left ventricular function and tomographic radionuclide perfusion: the Western Washington intracoronary streptokinase in myocardial infarction trial. Circulation 1984;70:867-75.

15 Stack RS, Phillips HR III, Grierson DS, et al. Functional improvement of jeopardized myocardium following intracoronary streptokinase infusion in acute myocardial infarction. $f$ Clin Invest 1983;72:84-95.

16 Leiboff RH, Katz RJ, Wasserman AG, et al. A randomized, angiographically controlled trial of intracoronary streptokinase in acute myocardial infarction. Am $\mathcal{f}$ Cardiol 1984;53:404-7.

17 Rogers WJ, Hood WP Jr, Mantle JA, et al. Return of left ventricular function after reperfusion in patients with myocardial infarction: importance of subtotal stenoses or intact collaterals. Circulation 1984;69:338-49.

18 Hooghoudt TEH, Serruys PW, Reiber JHC, Slager CJ, van den Brand M, Hugenholtz PG. The effect of recanalization of the occluded coronary artery in acute myocardial infarction on left ventricular function. Eur $\mathcal{F}$ Cardiol 1982;3:416-21.

19 Smalling RW, Fuentis F, Matthews MW, et al. Sustained improvement in left ventricular function and mortality by intracoronary streptokinase administration during evolving myocardial infarction. Circulation 1983;68:131-8.

20 Sheehan FH, Mathey DG, Schoffer J, Krebber H-J, Dodge HT. Effect of interventions in salvaging left ventricular function in acute myocardial infarction: a study of intracoronary streptokinase. Am f Cardiol 1983;52:431-8.

21 Wackers FJ, Berger HJ, Weinberg MA, Zaret BL. Spontaneous changes in left ventricular function over the first 24 hours of acute myocardial infarction: implications for evaluating early therapeutic interventions. Circulation 1982;66:748-54.

22 Sheehan FH, Mathey DG, Schofer J, Dodge HT, Bolson EL. Factors that determine recovery of left ventricular function after thrombolysis in patients with acute myocardial infarction. Circulation 1985;71:1121-8.

23 Marier DL, Gibson DG. Limitations of two frame method for displaying regional left ventricular wall motion in man. $\mathrm{Br}$ Heart I 1980;44:555-9.

24 Spann JF, Sherry S, Carabello BA, et al. Coronary thrombolysis by intravenous streptokinase in acute myocardial infarction: acute and follow-up studies. Am f Cardiol 1984;53:655-61.

25 Cribier A, Berland J, Champoud O, Moore N, Behar P, Letac B. Intracoronary thrombolysis in evolving myocardial infarction. Sequential angiographic analysis of left ventricular performance. Br Heart $\mathcal{F}$ 1983;50:401-10. 